\title{
Estimation of Saturation Flow Rate and Start-Up Lost Time for Signal Timing Based on Headway Distribution
}

\author{
Yi Zhao, ${ }^{1,2}$ Wenbo Zhang, ${ }^{3}$ Jian Lu, ${ }^{4}$ Wenjun Zhang, and Yongfeng Ma ${ }^{4}$ \\ ${ }^{1}$ Jiangsu Key Laboratory of Urban ITS, Southeast University, Si Pai Lou No. 2, Nanjing 210096, China \\ ${ }^{2}$ Jiangsu Province Collaborative Innovation Center of Modern Urban Traffic Technologies, Southeast University, \\ Si Pai Lou No. 2, Nanjing 210096, China \\ ${ }^{3}$ School of Civil Engineering, Purdue University, 550 Stadium Drive, West Lafayette, IN 47907, USA \\ ${ }^{4}$ School of Transportation, Southeast University, Si Pai Lou No. 2, Nanjing 210096, China \\ Correspondence should be addressed to Jian Lu; lujian_1972@seu.edu.cn
}

Received 5 May 2015; Revised 31 July 2015; Accepted 2 August 2015

Academic Editor: Rigoberto Medina

Copyright (C) 2015 Yi Zhao et al. This is an open access article distributed under the Creative Commons Attribution License, which permits unrestricted use, distribution, and reproduction in any medium, provided the original work is properly cited.

\begin{abstract}
This study aimed to calibrate saturation flow rate (SFR) and start-up lost time (SLT) when developing signal timing. In current commonly used methods, SFR for one given lane is usually calibrated from many subjective adjustment factors and a fixed result. SLT is calculated based on the fixed SFR, which prevents local applications in China. Considering the importance of traffic behavior (headway) in determining SFR and SLT, this study started from headway distribution and attempted to specify the relationships between headway and vehicle position directly. A common intersection in Nanjing, China, was selected to implement field study and data from 920 queues was collected. Headway distribution was explored and the 78th percentile of headway at each position was selected to build model. Based on the developed relationships, SFR and SLT were calibrated. The results showed that SFR and SLT were correlated with queue length. Moreover, the results showed that it was difficult to reach saturated state even with a long queue length. This paper provides a new perspective on calibrating important parameters in signal timing, which will be useful for traffic agencies to complete signal timing by making the process simpler.
\end{abstract}

\section{Introduction}

Signalized intersections are widely used in different roads in many areas around the world. In general, these traffic light signals are seen as feasible and efficient tools to guarantee the efficiency and safety of intersections, especially on city roads and arterial roads. Maintaining reasonable signal timing is necessary, but difficult. Sensible signal timing can maintain order among all vehicles on all lanes and directions and reduce potential conflict points. If excessive time is allocated, the traffic light will remain green after all vehicles have passed the intersection. This is a big waste which can greatly reduce a highway's capacity and level of service (LOS). Green signals that are too short will lead to frequent stops and starts, and lanes or certain directions would be crowded with vehicles during peak hours. More importantly, the intersection is not an isolated point but an important node in the traffic network.
Poor LOS of an intersection caused by suboptimal signal timing will extend and influence downstream intersections, effectively reducing the LOS of the whole network.

After the implementation of traffic signals, many organizations and scholars developed methods for signal timing. Some examples include TRANSYT in Britain, Webster and ARRB in Australia, Highway Capacity Manual (HCM) in America, and stop line method and conflict point method in China [1-3]. TRANSYT tries to optimize static signal timing through traffic simulation. ARRB, an extension of Webster, is an algorithm based on traffic saturation flow, which is determined by lanes and environment. In China, some scholars also developed some unique methods of signal timing. The stop line method and the conflict point method are both based on highway capacity but differ in computing. The stop line method computes capacity for each special lane based on headway. The conflict point method introduces 
a number of conflict points which will influence highway capacity. HCM (2010) also provides a common and detailed method for signal timing, considering saturation flow rate (SFR) and start-up lost time (SLT), as shown in (1)-(3). In these equations, traffic demand $v_{i}$ can be obtained through field study and volume-to-capacity ratio $X_{i}$ is determined according to the traffic agency, usually equal to 1.00 . The more complicated computation is SFR, as shown in (1). Many subjective adjustment factors are introduced, which in turn increase the risk of errors. HCM (2010) also proposes that after the fourth vehicle of a queue, vehicles will own stable and similar headways. The average headway of vehicles after the fourth vehicle is used to compute saturation flow rate. However, the exception is SLT, which is also introduced to measure the differences in headways of the 1st to 5 th vehicles and saturated vehicles:

$$
\begin{aligned}
s & =s_{0} N f_{w} f_{\mathrm{HV}} f_{g} f_{p} f_{b b} f_{\mathrm{RT}} f_{\mathrm{LT}}, \\
C & =\frac{L X_{c}}{X_{c}-\sum_{i \in c i} y_{c, i}}, \\
g_{i} & =\frac{v_{i} C}{N_{i} s_{i} X_{i}}=\left(\frac{v}{N s}\right)_{i}\left(\frac{C}{X_{i}}\right),
\end{aligned}
$$

where $s$ : SFR; $s_{0}$ : basic SFR under ideal conditions; $f$ : adjustment factors; $C$ : cycle length (s); L: cycle lost time (s); $X_{c}$ : critical intersection volume-to-capacity ratio; $y_{c, i}:$ critical flow ratio for phase $i, v_{i} /\left(N s_{i}\right) ; c_{i}$ : critical phases on the critical path; $X_{i}$ : volume-to-capacity ratio for phase $i ; v_{i}$ : demand flow rate for phase $i$ (veh./h); $N_{i}$ : number of lanes for phase $i(\ln )$; $s_{i}$ : saturation flow rate for phase $i$ (veh./h/ln); and $g_{i}$ : effective green time for phase $i$ (s).

Despite the availability of various methods, it is still a difficult problem for local traffic agency to develop signal timing due to the many adjustment factors. Each intersection may own special characteristics, that is, geometry, access categories, heavy traffic percentage, highway maintenance, and traffic behaviors. These factors will influence the values of adjustment factors and highway capacity [4]. Unfortunately, some characteristics may not even be included in recommendations of existing methods. Hence, there is confusion on how to measure these factors. Even for the same intersection, traffic will vary across time. Dynamic signal timing is a better option but cannot be processed by the above methods. Thus, finding an easy way to calibrate important factors, that is, SFR and SLT, is necessary and meaningful.

HCM (2010) recommends default values for SFR. Moreover, the manual also suggests that for different areas the agency should calibrate the factor based on field study. Zhang and Chen [5] validated the method in China and concluded that SFR should be calibrated specially. To measure flow characteristics, many scholars focused on traffic behaviors, namely, discharge headway [6]. Furthermore, some external factors, such as the number of lanes and vehicle types, are also introduced to obtain accurate departure headways. Gerlough and Wagner [7] established the technical report "New Criteria for Designing and Timing Traffic Signals." King and Wilkinson [8] made measurements to determine the relative effectiveness of various signal configurations and lengths sizes in dissipating queues. Hossain [9] introduced a new microscopic simulation technique to estimate the saturation flow from the influencing variables like road width, turning proportion, and percentage of heavy and nonmotorized vehicles. Li and Wang [10] present a practical vehicle waiting time estimation method using an adaptive video-based vehicle tracking method and the results of experiments show the effectiveness of the proposed approach. The common conclusion on discharge headway is that the values are correlated to the queue position. In general, the first vehicle owns the largest departure headway and the values decrease with the increase in position. After the 4 th or 5 th vehicle, the value becomes similar with no significant differences. Thus, some applications adopted the average headway of 4 th to 10 th/12th vehicles as the saturation headway and computed saturation flow rate based on that $[11,12]$. However, recent studies show that using just the average of saturated discharge headways to calculate the SFR may result in underestimations [13-15]. The SLT is the sum of differences between the headways of the first four or five vehicles and saturated headways, and little literature focuses on the topic. In general, HCM (2010) recommends $2 \mathrm{~s}$ as the default value of SLT if signalized. Above all, though the relationship between headway and vehicle position has been verified, there are few mathematical forms to specify them. Therefore, it is difficult to calibrate factors and develop signal timing, especially for dynamic signal timing.

In the paper, an analytical method is developed to calibrate SFR and SLT based on discharge headway distribution. An intersection in Nanjing, China, is selected to obtain discharge headways and used as a case study. Mathematical forms are developed to specify the relationship between headway and vehicle position. Further, SFR and SLT are determined under different queue lengths (corresponding to different traffic volumes) based on these relationships. At the same time, there are several limitations with this paper that need to be strengthened in future study, such as no consideration on the proportion of heavy traffic and on lane sharing (left/trough movements).

The rest of this paper is organized as follows. In the second section, the field study and data statistics are summarized. The third section analyzes the headway distribution and the relationship between headway and vehicle position is developed. In the fourth part, SFR and SLT are calibrated. The final section presents the conclusion and recommendations for future study. The technological process is shown in Figure 1.

\section{Data Collection}

A common intersection in Nanjing, China, was selected to complete the field study. The two roads that cross at the intersection are Xinglong Street and Jiangdong Road, and both are arterial roads. Figure 2 shows the detailed channelization of the intersection. There are three through lanes, one left turn lane and one right turn lane in each approach. The intersection is located in the central part of the city with rather high traffic volumes, so signal control is now 


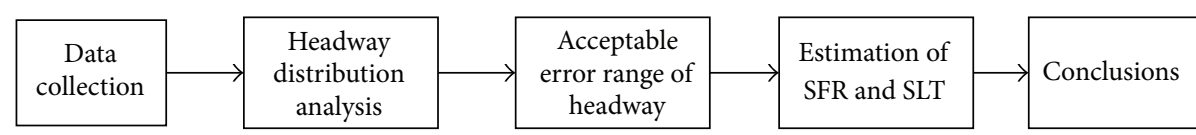

FIGURE 1: The flowchart of this paper.
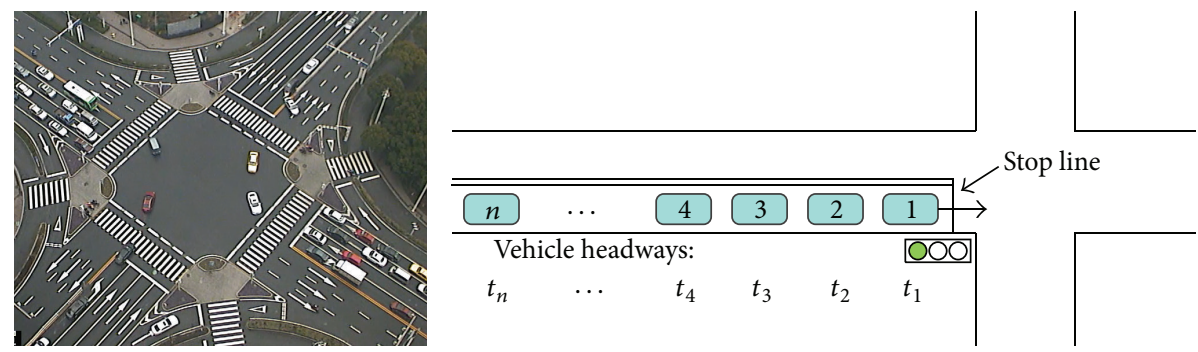

FIGURE 2: Collection site and headways at the intersection.

adopted. The timing scheme in morning peak hours contains four phases, and the cycle time is $134 \mathrm{~s}$.

The field study focused on straight traffic on the eastbound approach during morning peak and evening peak times on weekdays. Video cameras were used to record the real traffic conditions around the intersection. With the help of video-editing software, all recorded videos were processed manually. More importantly, to improve the accuracy of data collection, discharge headways were defined before counting. For the first vehicle of a queue, the discharge headway was the elapsed time between the start of a green light and the time when the vehicle's front bumper passed the stop line. For the remaining vehicles in the queue, including all the vehicles that join the queue during the green light, the discharge headways were the elapsed time between the times when two successive vehicles' front bumpers passed the same stop line. Considering the weak role in specifying headway distribution, queues with less than five vehicles were excluded. However, queues with weaving vehicles and queues influenced by pedestrians, drivers' distractions, or other reasons were under survey, since they mirror actual driving behavior. In total, the data from 920 groups of queued vehicles was obtained and the corresponding statistics were summarized in Table 1 (one cycle is seen as one group). The lengths of all 920 groups were not all the same: 817 groups reached 10 vehicles while only 26 groups reached 20 vehicles. As such, there were fewer observed vehicles in far back positions.

\section{Discharge Headway Distribution}

Figure 3 presents the headway distribution across vehicle positions. The headways have a significant decreasing tendency with the increase of vehicle position in a queue, though with some minor fluctuations at the beginning and at the end. Directly developing models with all headways is difficult and may cause inaccuracies. Thus, six important statistical values at each position in queue, the $95 \%$ percentile, $85 \%$ percentile, $75 \%$ percentile, $65 \%$ percentile, and $50 \%$ percentile, are extracted and graphed in Figure 2. The tendencies of these six
TABLE 1: Statistical parameters of entering headway (unit of headway: second).

\begin{tabular}{lccccc}
\hline Veh. pos. & Valid group & Min & Max & Mean & Std. dev. \\
\hline 1 & 920 & 0.80 & 10.00 & 2.56 & 0.46 \\
2 & 920 & 1.64 & 7.00 & 3.28 & 0.34 \\
3 & 920 & 1.04 & 7.36 & 2.80 & 0.34 \\
4 & 920 & 1.16 & 6.72 & 2.56 & 0.31 \\
5 & 920 & 1.24 & 5.64 & 2.44 & 0.29 \\
6 & 916 & 1.20 & 5.12 & 2.40 & 0.28 \\
7 & 908 & 1.08 & 6.32 & 2.36 & 0.29 \\
8 & 890 & 1.12 & 6.28 & 2.28 & 0.29 \\
9 & 874 & 1.04 & 5.40 & 2.24 & 0.26 \\
10 & 817 & 1.08 & 5.56 & 2.12 & 0.26 \\
11 & 721 & 1.02 & 5.20 & 2.08 & 0.22 \\
12 & 655 & 1.12 & 4.76 & 2.04 & 0.21 \\
13 & 572 & 1.04 & 6.00 & 2.00 & 0.21 \\
14 & 488 & 1.00 & 4.44 & 1.92 & 0.19 \\
15 & 346 & 1.12 & 4.96 & 1.92 & 0.19 \\
16 & 205 & 1.20 & 3.84 & 1.92 & 0.18 \\
17 & 110 & 1.16 & 3.44 & 1.80 & 0.16 \\
18 & 65 & 1.24 & 2.76 & 1.88 & 0.15 \\
19 & 41 & 1.16 & 2.92 & 1.80 & 0.14 \\
20 & 26 & 1.32 & 2.68 & 1.84 & 0.16 \\
\hline
\end{tabular}

values similarly decrease and also indicate the first headways under each value are not in accordance with others.

Considering the distribution in Figure 3, several mathematical forms were used to fit the headway distribution rule, and the logarithmic form was found to be better at specifying relationships between headway and vehicle positions. Here $y$ denotes the headways and $x$ refers to vehicle position. The logarithmic forms under different percentiles are developed and shown in Table 2. The metric of model fit, $R^{2}$, is also presented for each percentile. If including the first vehicle, $R^{2}$ is found to be around 0.8 . However, if excluding the fluctuations caused by the first vehicle, the model fit improves 
TABLE 2: Headway distribution in different percentiles.

\begin{tabular}{lccc}
\hline Percentiles & Logarithmical forms (exclude the first vehicle) & $R^{2}$ (exclude the first vehicle) & $R^{2}$ (for the whole queue) \\
\hline Mean & $y=-0.472 \ln (x-1)+3.17$ & 0.9842 & 0.7906 \\
95th per. & $y=-0.973 \ln (x-1)+5.32$ & 0.9321 & 0.8605 \\
85th per. & $y=-0.676 \ln (x-1)+4.13$ & 0.9619 & 0.8789 \\
75th per. & $y=-0.572 \ln (x-1)+3.65$ & 0.9772 & 0.8633 \\
65th per. & $y=-0.507 \ln (x-1)+3.35$ & 0.9778 & 0.8021 \\
50th per. & $y=-0.444 \ln (x-1)+3.02$ & 0.9787 & 0.7207 \\
\hline
\end{tabular}

${ }^{*} x$ is an integer from 2 to 20.

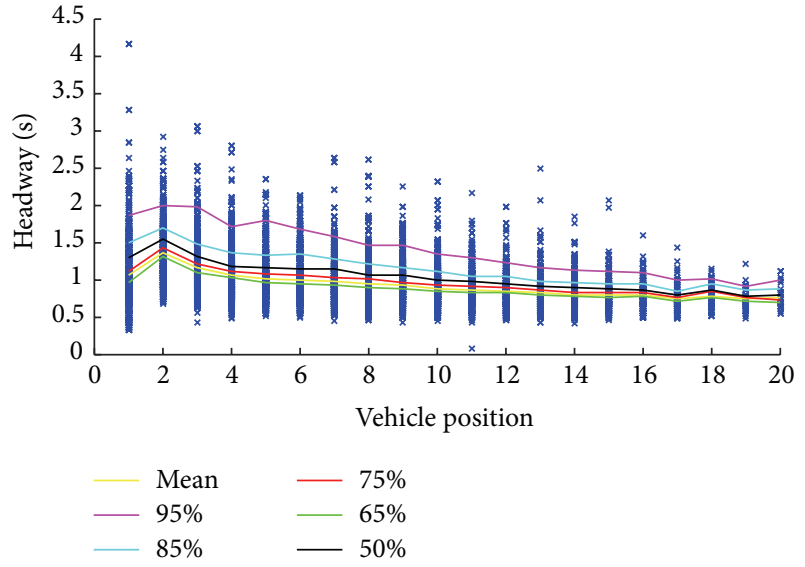

FIGURE 3: Headway distribution across vehicle position.

greatly (more than 0.95 ). Thus, to obtain a more accurate logarithmic form, the headways of the first vehicle in each cycle were put aside.

With known logarithmical forms, the total time needed, in theory, can be obtained through the sum of all vehicles' headway. If there are $n$ vehicles in one queue, the headway of each vehicle can be computed with $f$. The assigned green time will be equal to the total headways of all $n$ vehicles to guarantee all vehicles can pass the stop line when the light is green. The mathematical forms are presented as follows:

$$
T_{G}=\sum_{i=1}^{n} y_{i}=\sum_{i=1}^{n} f(i) \text {, }
$$

where $T_{G}$ : green time assigned for queue vehicles; $y_{i}$ : headway of vehicles at different positions; $f$ : relationship between headway and vehicle position; $i$ : vehicle position; and $n$ : queue length.

The next problem is deciding which percentile will be better to compute the assigned green time. If higher percentiles are used, it is obvious that nearly all vehicles in the queue can pass the stop line during a green light. However, the risks of wasting green time (no vehicles in queue toward the end of the green light) would increase. If a lower percentile is used, another problem emerges. Most vehicles cannot pass the stop line during one green light and must wait for the second or third green light. This will greatly reduce traffic efficiency around the intersection, even in regional networks. Thus, one rule should be designed to determine the appropriate percentile to use.

The primary purpose of signal timing is to guarantee that queued vehicles pass the stop line within one cycle. The probability of realizing the purpose can be treated as the rule to determine appropriate percentile. However, no literature or relevant specification has ever suggested any reasonable value for the probability. Further study can be conducted on this, but it is beyond the scope of this paper. In this paper, $75 \%$ was recommended as the minimum probability. This value should be optimized in the future.

A metric, $T_{A}$, is introduced to measure the total real time of each queue spent on passing the stop line. If $T_{G} \geq T_{A}$, it means the green time is long enough for queues to pass the stop line during one cycle. In contrast, $T_{G}<T_{A}$, which means queues fail to pass the stop line during one cycle. The probabilities of queues passing the stop line during one cycle under different percentiles are presented in Table 3. From Table 3, we can see that selecting higher percentile values to specify logarithmic forms allows more queues to pass the stop line during one cycle. Meanwhile, with more vehicles in a queue, the probability of the queue passing the stop line during one cycle will also be high.

Table 3 also shows that the minimum probabilities of queues passing the stop line are $97 \%, 86 \%, 75 \%, 71 \%, 59 \%$, and $42 \%$ when statistical values at each position in the queue are, respectively, $95 \%$ percentile, $85 \%$ percentile, $78 \%$ percentile, $75 \%$ percentile, $65 \%$ percentile, and $50 \%$ percentile. There is no doubt that only the $78 \%$ percentile can well meet the purpose of signal timing. The corresponding logarithmic form of headway distribution is $y=-0.600 \ln (x-1)+3.79$, with $R^{2}$ of 0.9761 .

\section{SFR and SLT Calibration}

4.1. Influence of Queue Length. Before calibrating SFR and SLT using developed relationships, there is an unsolved problem: the influence of queue length. In general, the queue's discharge will be seen as stable after the fourth vehicle, as recommended by HCM (2010). In other words, there are no significant differences in flow ratio after the fourth vehicle in the queue and the flow is saturated. In this section, we validate the influence of queue length by comparing SFR among vehicles at different positions.

The acceptable errors in SFR are introduced based on acceptable errors in critical flow ratio. If the difference in SFR between two vehicles at different positions is more than 
TABLE 3: Probabilities of queues passing stop line during one cycle.

\begin{tabular}{|c|c|c|c|c|c|c|c|c|}
\hline Queue length & Valid group & Mean & 95th per. & 85th per. & 78th per. & 75th per. & 65th per. & 50th per. \\
\hline 2 & 920 & $51.2 \%$ & $97.4 \%$ & $86.0 \%$ & $75.8 \%$ & $71.7 \%$ & $59.7 \%$ & $42.0 \%$ \\
\hline 3 & 920 & $52.7 \%$ & $99.0 \%$ & $90.4 \%$ & $81.2 \%$ & $76.1 \%$ & $64.0 \%$ & $43.8 \%$ \\
\hline 4 & 920 & $56.4 \%$ & $99.5 \%$ & $93.5 \%$ & $87.1 \%$ & $81.1 \%$ & $66.0 \%$ & $43.6 \%$ \\
\hline 5 & 920 & $55.3 \%$ & $99.6 \%$ & $95.5 \%$ & $87.6 \%$ & $82.8 \%$ & $68.2 \%$ & $45.3 \%$ \\
\hline 6 & 920 & $54.6 \%$ & $99.8 \%$ & $96.3 \%$ & $87.4 \%$ & $81.4 \%$ & $69.9 \%$ & $43.2 \%$ \\
\hline 7 & 916 & $56.9 \%$ & $99.3 \%$ & $95.9 \%$ & $88.4 \%$ & $82.2 \%$ & $67.2 \%$ & $43.6 \%$ \\
\hline 8 & 908 & $56.1 \%$ & $99.6 \%$ & $95.6 \%$ & $88.0 \%$ & $83.4 \%$ & $67.7 \%$ & $44.2 \%$ \\
\hline 9 & 890 & $56.6 \%$ & $99.8 \%$ & $94.4 \%$ & $86.7 \%$ & $82.1 \%$ & $68.8 \%$ & $42.2 \%$ \\
\hline 10 & 834 & $58.0 \%$ & $100.0 \%$ & $94.2 \%$ & $89.6 \%$ & $86.2 \%$ & $71.8 \%$ & $45.4 \%$ \\
\hline 11 & 742 & $62.0 \%$ & $100.0 \%$ & $96.5 \%$ & $89.9 \%$ & $87.3 \%$ & $75.5 \%$ & $48.4 \%$ \\
\hline 12 & 664 & $62.0 \%$ & $100.0 \%$ & $97.9 \%$ & $92.5 \%$ & $88.7 \%$ & $77.4 \%$ & $49.4 \%$ \\
\hline 13 & 582 & $66.8 \%$ & $100.0 \%$ & $97.9 \%$ & $94.0 \%$ & $91.1 \%$ & $80.2 \%$ & $53.1 \%$ \\
\hline 14 & 496 & $67.9 \%$ & $100.0 \%$ & $98.0 \%$ & $93.5 \%$ & $91.7 \%$ & $80.4 \%$ & $54.2 \%$ \\
\hline 15 & 348 & $68.7 \%$ & $100.0 \%$ & $98.9 \%$ & $94.8 \%$ & $92.5 \%$ & $81.3 \%$ & $53.2 \%$ \\
\hline 16 & 206 & $68.0 \%$ & $100.0 \%$ & $98.5 \%$ & $95.6 \%$ & $94.2 \%$ & $80.1 \%$ & $52.9 \%$ \\
\hline 17 & 109 & $65.1 \%$ & $100.0 \%$ & $98.2 \%$ & $94.5 \%$ & $91.7 \%$ & $78.0 \%$ & $51.4 \%$ \\
\hline 18 & 65 & $58.5 \%$ & $100.0 \%$ & $96.9 \%$ & $92.3 \%$ & $89.2 \%$ & $69.2 \%$ & $49.2 \%$ \\
\hline 19 & 41 & $65.9 \%$ & $100.0 \%$ & $95.1 \%$ & $95.1 \%$ & $92.7 \%$ & $78.0 \%$ & $61.0 \%$ \\
\hline 20 & 26 & $61.5 \%$ & $100.0 \%$ & $100.0 \%$ & $96.2 \%$ & $92.3 \%$ & $73.1 \%$ & $50.0 \%$ \\
\hline
\end{tabular}

the acceptable errors, we suggest that the vehicle position will influence the SFR. In contrast, when there are no significant influences of vehicle positions, the flow can be seen as saturated:

$$
\Delta s=s-\frac{q}{\left(q / s+\theta^{*}\right)}
$$

where $\Delta s$ : errors in SFR, veh./h/ln; $s$ : SFR, veh./h/ln; $\theta^{*}$ : the acceptable errors in critical flow ratio; and $q$ : traffic demand, veh./h/ln.

In (5), the acceptable errors in critical flow ratio, $\theta^{*}$, are very important. The changes in cycle length under different errors in critical flow ratio are introduced to measure acceptable errors. Based on HCM (2010), cycle length $(C)$ is determined by start-up lost time $(L)$ and the summation of critical flow ratio $(Y)$, as shown in (6). $Y$ is also defined as the intersection's saturation degree. Under different critical flow ratios and changes, the changes in assigned cycle length are also computed and presented in Figure 4:

$$
R(Y, \theta)=\frac{C_{Y+\theta}-C_{Y}}{C_{Y+\theta}}=1-\frac{L /(1-Y)}{L /(1-Y+\theta)}=\frac{\theta}{1-Y},
$$

where $R(Y, \theta)$ : the change rate of cycle length; $C_{Y+\theta}, C_{Y}$ : cycle lengths; $L$ : total lost time; $Y$ : summation of critical flow ratio; and $\theta$ : the errors in critical flow ratio.

According to "Code for Planning of Intersection on Urban Road" (GB 50647-2011 in China), changes in cycle length which are less than $5 \%$ can be ignored. From Figure 3, the changes in cycle length are less than $5 \%$ when the errors, $\theta$, are less than 0.01 . Thus, the acceptable errors in critical flow ratio, $\theta^{*}$, can be equal to or less than 0.01 . Substituting the value into (5), the threshold of acceptable errors in SFR can be obtained, as shown in Figure 5. In Figure 5, the surface

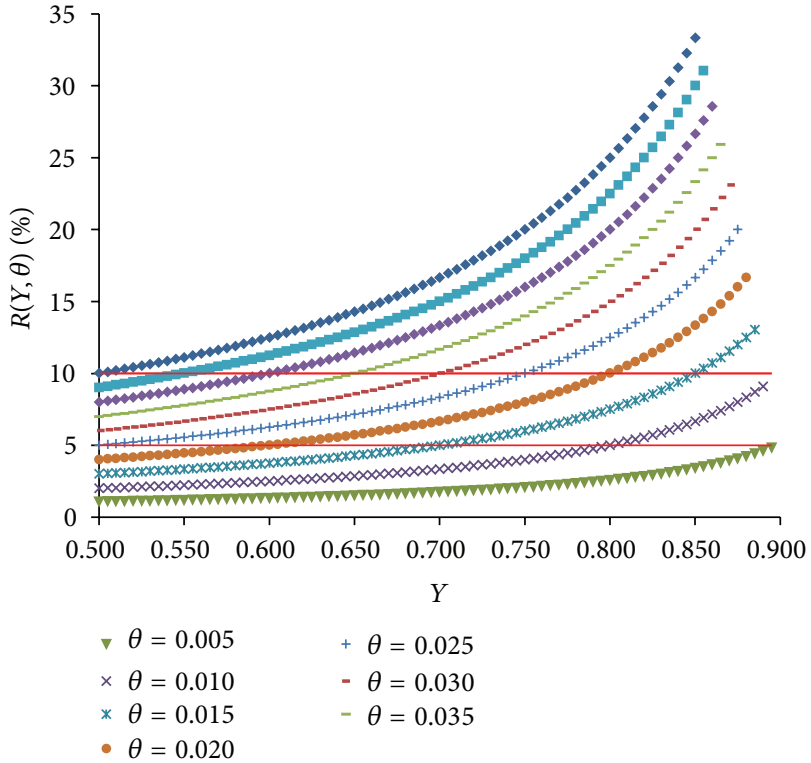

FIGURE 4: Change rate of cycle length with the change of saturation degree.

presents the changes in SFR under the threshold of errors in critical flow ratio of 0.01 . Under the surface, the errors in critical flow ratio are less than 0.01 , and, above the surface, the errors are more than 0.01 . It can be concluded that most errors in critical flow ratio are less than 0.01 when the change in SFR is less than 60. Thus, the threshold of errors in SFR is $60 \mathrm{veh} . / \mathrm{h} / \mathrm{ln}$.

4.2. SFR Calibration. With the relationship between headway and vehicle position (under the 78th percentile: 


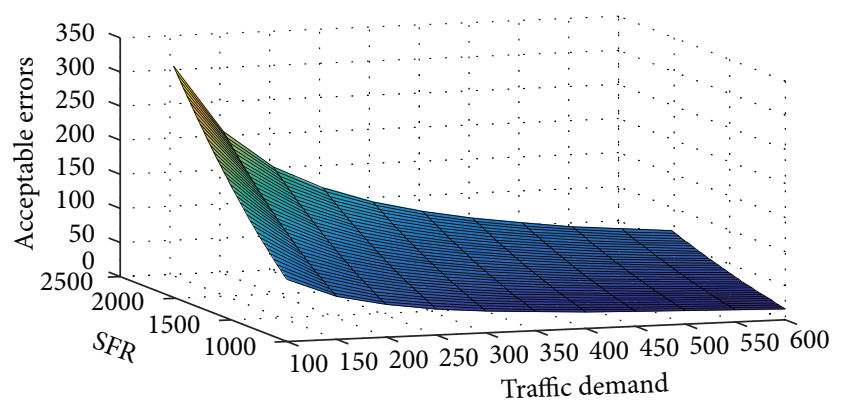

FIGURE 5: The changes in SFR under threshold of errors in critical flow ratio.

TABLE 4: SFR at different vehicle positions.

\begin{tabular}{lccc}
\hline Queue length & $\begin{array}{c}\text { Saturated } \\
\text { headway (s) }\end{array}$ & $\begin{array}{c}\text { SFR } \\
\text { (veh./h/ln) }\end{array}$ & $\begin{array}{c}\text { Differences in SFR } \\
\text { with prior vehicle } \\
\text { (veh./h/ln) }\end{array}$ \\
\hline 1 & - & - & - \\
2 & 3.80 & 951 & - \\
3 & 3.36 & 1068 & 117 \\
4 & 3.12 & 1151 & 83 \\
5 & 2.96 & 1219 & 67 \\
6 & 2.80 & 1277 & 58 \\
7 & 2.72 & 1328 & 52 \\
8 & 2.60 & 1375 & 47 \\
9 & 2.52 & 1419 & 43 \\
10 & 2.48 & 1459 & 41 \\
11 & 2.40 & 1498 & 38 \\
12 & 2.36 & 1534 & 37 \\
13 & 2.28 & 1569 & 35 \\
14 & 2.24 & 1603 & 34 \\
15 & 2.20 & 1635 & 32 \\
16 & 2.16 & 1667 & 31 \\
17 & 2.12 & 1697 & 30 \\
18 & 2.08 & 1727 & 30 \\
19 & 2.04 & 1755 & 29 \\
20 & 2.00 & 1784 & 28 \\
\hline & & &
\end{tabular}

$y=-0.600 \ln (x-1)+3.79)$, corresponding flow rate (veh./h/ln) at different vehicle positions can be calculated, as shown in Table 4. From Table 4, the difference in SFR of queues with five vehicles and 20 vehicles is $565 \mathrm{veh} . / \mathrm{h} / \mathrm{ln}$, which is larger than the threshold of acceptable errors in SFR. Though some differences in SFR between two successive queue lengths are less than 60, many low differences in SFR are found between two successive queues with odd or even vehicles. For example, the difference in SFR between the queues with 19 vehicles and 20 vehicles is $28 \mathrm{veh} . / \mathrm{h} / \mathrm{ln}$, while the difference in SFR between the queues with 16 vehicles and 18 vehicles is $60 \mathrm{veh} . / \mathrm{h} / \mathrm{ln}$. Thus, the effects of vehicle positions on SFR should be considered.

Table 4 also presents the SFR values for different queue lengths, for example, $1219 \mathrm{veh} . / \mathrm{h} / \mathrm{ln}, 1459 \mathrm{veh} . / \mathrm{h} / \mathrm{ln}$,
$1635 \mathrm{veh} . / \mathrm{h} / \mathrm{ln}$, and $1784 \mathrm{veh} . / \mathrm{h} / \mathrm{ln}$ for queues with only 5 vehicles, 10 vehicles, 15 vehicles, and 20 vehicles, respectively. Higher SFR may happen when the queue length is longer. Hence, it is necessary to make accurate predictions about queue length in order to obtain accurate SFRs. Furthermore, the errors of predicted queue length should not exceed two vehicles when the queue length is more than 6 .

4.3. SLT Calibration. SLT is the sum of the differences between unsaturated headways and saturated headways. In this paper, considering the effects of queue length on SFR, the headway of the last vehicle in a queue is seen as saturated headway. The SLT can be calibrated based on headway at different positions and saturated headway, as shown in (7). The SLT is correlated to queue length. The more the vehicles in a queue are, the higher the SLT will be. For example, SLTs are $1.7 \mathrm{~s}, 3 \mathrm{~s}$, and $8.1 \mathrm{~s}$ for queues with only 5 vehicles, 7 vehicles, and 15 vehicles, respectively:

$$
\begin{aligned}
\text { SLT } & =\sum_{x=1}^{n}[f(x)-H], \\
H & =f(n),
\end{aligned}
$$

where $x$ : vehicle position; $H$ : saturated headway; and $n$ : the position of the last vehicle in one queue.

\section{Conclusions}

The main objective of this study is to calibrate saturation flow rate (SFR) and start-up lost time (SLT). The field study and case study are both located in a common intersection in Nanjing from where data from 920 queues was collected. Logarithmic forms are developed to specify relationships between headway and vehicle positions based on headway distribution. To seek a balance between high passing probability and fewer instances of wasted green signal times, the study reached a conclusion using the 78th percentile of headways at each position to build model.

Before calibrating SFR and SLT, the accepted errors in SFR are debated to validate the effects of queue length on SFR and $60 \mathrm{veh} . / \mathrm{h} / \mathrm{ln}$ is determined as the ideal threshold. SFRs are measured based on developed logarithmic forms. Comparing the differences in SFR among different queue lengths, it is difficult to obtain the saturated state when the queue length is less than 20. Moreover, the effects of queue length on SFR cannot be ignored. Therefore, SFR should be calibrated considering queue length. For example, the values of SFR for the observed lane were 1219 veh./h, 1459 veh./h, 1635 veh./h, and $1784 \mathrm{veh}$./h for queues with 5 vehicles, 10 vehicles, 15 vehicles, and 20 vehicles, respectively. SLT should also be calibrated based on SFR and developed logarithmic forms, with the assumption that the headway of the last vehicle is the saturated headway. Again, queue length is a necessary and important variable in calibrating SFR and SLT.

Obviously, there is still one unsolved problem, which can be solved in the future. Due to limited data resources, only one lane was discussed in this study. To obtain more detailed and accurate headway distribution, the field study should be 
implemented at more similar intersections. Data should also be observed on weekends, when potential temporal variances may occur.

\section{Conflict of Interests}

The authors declare that there is no conflict of interests regarding the publication of this paper.

\section{Acknowledgment}

This work is sponsored by National Key Technology Support Program. The Program number is 2014BAG01B06.

\section{References}

[1] M. R. Crabtree, “TRANSYT/9 user's manual," TRRL Application Guide 8, Transport and Road Research Laboratory, Crowthorne, UK, 1988.

[2] Highway Capacity Manual 2010, Transportation Research Board, National Research Council, Washington, DC, USA, 2010.

[3] P. Q. Tan, Y. Zhao, and Y. Chao, "Signal-planning optimal model for intersection," Journal of Shanghai Tiedao University, vol. 20, no. $4,1999$.

[4] A. S. Al-Ghamdi, "Entering headway for through movements at urban signalized intersections," Transportation Research Record, vol. 1678, no. 1, pp. 42-47, 1999.

[5] G. Zhang and J. Chen, "Study on saturation flow rates for signalized intersections," in Proceedings of the International Conference on Measuring Technology and Mechatronics Automation (ICMTMA '09), pp. 598-601, IEEE, Zhangjiajie, China, April 2009.

[6] B. D. Greenshields, D. Schapiro, and E. L. Ericksen, Traffic Performance at Urban Street Intersections, Eno Foundation for Highway Traffic Control, 1947.

[7] D. L. Gerlough and F. A. Wagner, "Improved criteria for traffic signals at individual intersections," Highway Research Board NCHRP 32, National Research Council, Washington, DC, USA, 1967.

[8] G. F. King and M. Wilkinson, "Relationship of signal design to discharge headway, approach capacity, and delay," Transportation Research Record, no. 615, pp. 37-44, 1977.

[9] M. Hossain, "Estimation of saturation flow at signalised intersections of developing cities: a micro-simulation modelling approach," Transportation Research Part A: Policy and Practice, vol. 35, no. 2, pp. 123-141, 2001.

[10] L. Li and F.-Y. Wang, "Approximate vehicle waiting time estimation using adaptive video-based vehicle tracking," in Advances in Machine Vision, Image Processing, and Pattern Analysis, vol. 4153 of Lecture Notes in Computer Science, pp. 105-114, Springer, 2006.

[11] Transportation Research Board, Highway Capacity Manual 2000, National Research Council, Washington, DC, USA, 2000.

[12] H. Li and P. D. Prevedouros, "Detailed observations of saturation headways and start-up lost times," Transportation Research Record, no. 1802, pp. 44-53, 2002.

[13] A. Al-Ghamdi, "Entering headway for through movements at urban signalized intersections," Transportation Research Record, vol. 1678, pp. 42-47, 1999, Paper No. 99-1232.
[14] W. Wang, W. Zhang, H. Guo, H. Bubb, and K. Ikeuchi, "A safety-based approaching behavioural model with various driving characteristics," Transportation Research Part C: Emerging Technologies, vol. 19, no. 6, pp. 1202-1214, 2011.

[15] X. Jin, Y. Zhang, F. Wang et al., "Departure headways at signalized intersections: a log-normal distribution model approach," Transportation Research Part C: Emerging Technologies, vol. 17, no. 3, pp. 318-327, 2009. 


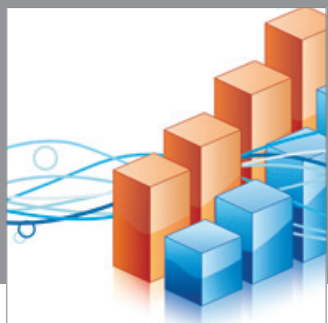

Advances in

Operations Research

mansans

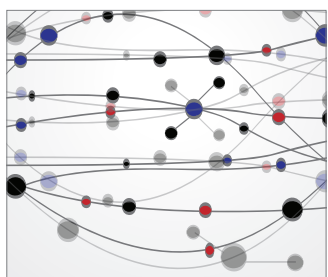

The Scientific World Journal
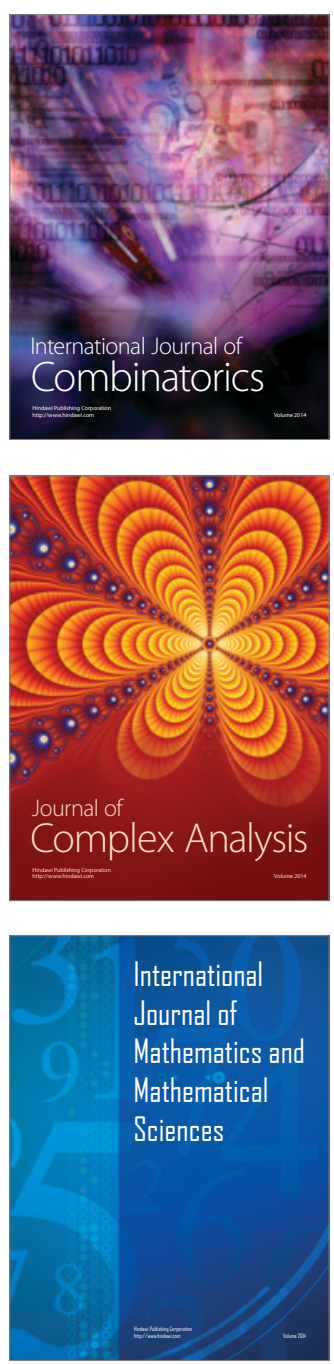
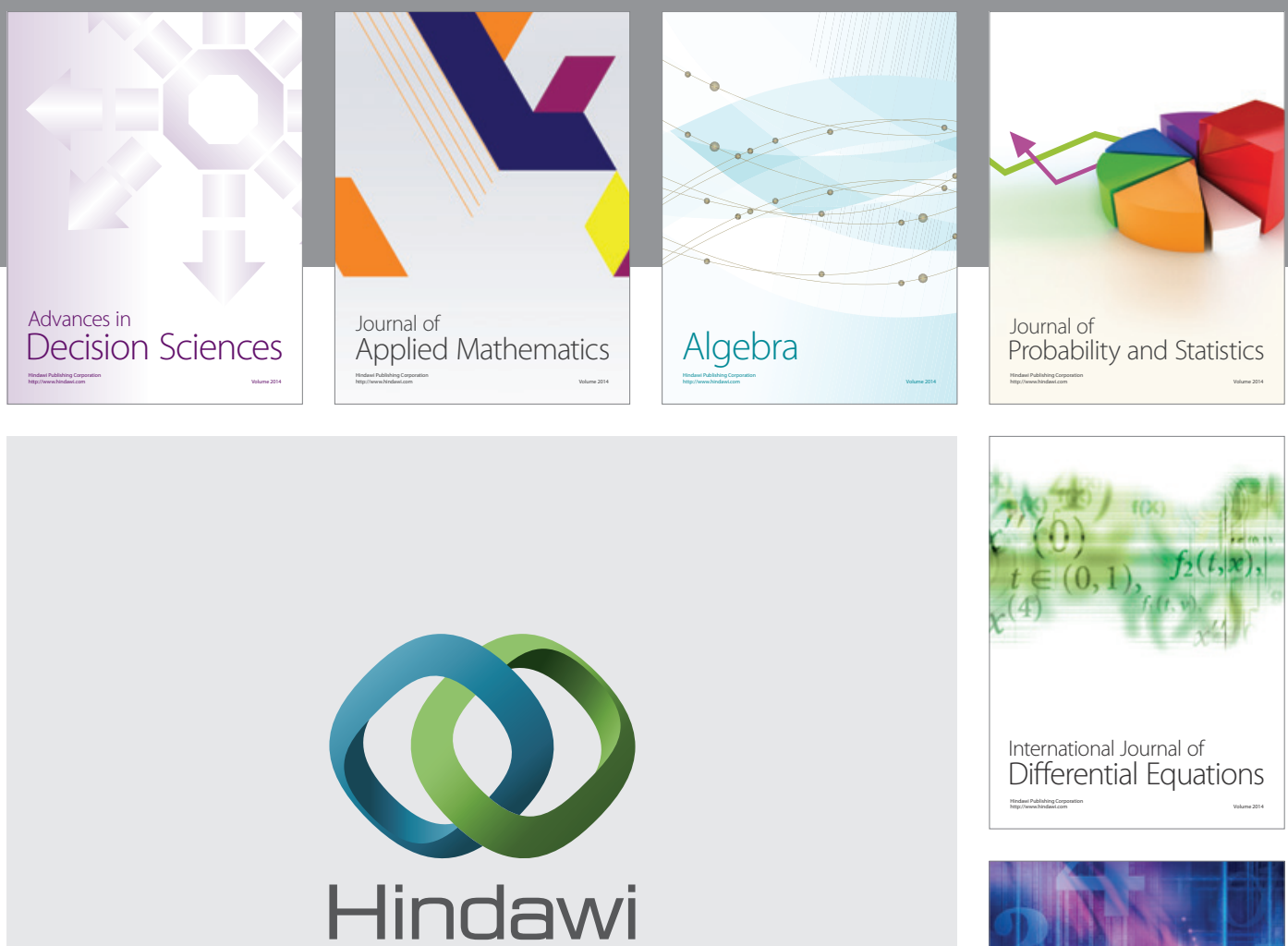

Submit your manuscripts at http://www.hindawi.com
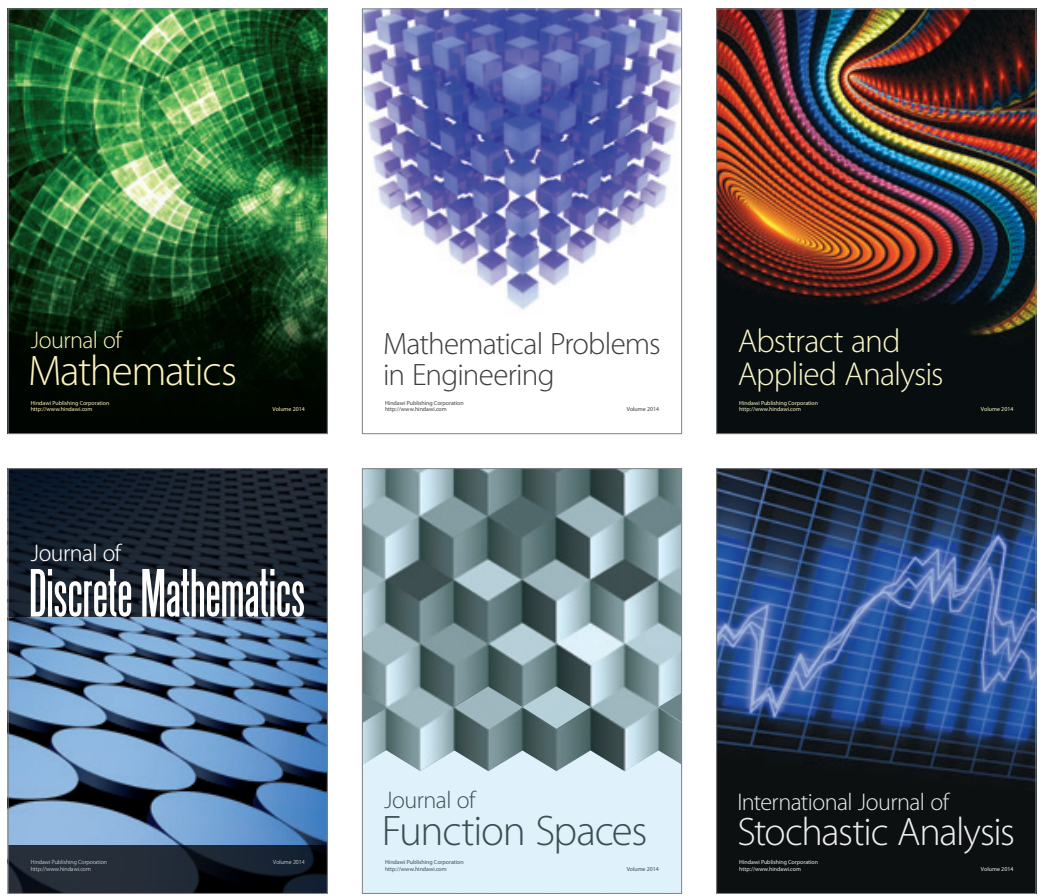

Journal of

Function Spaces

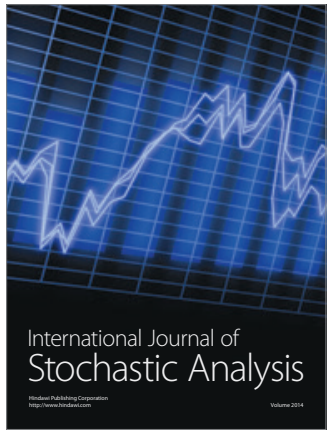

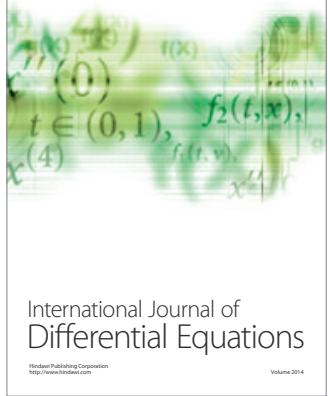
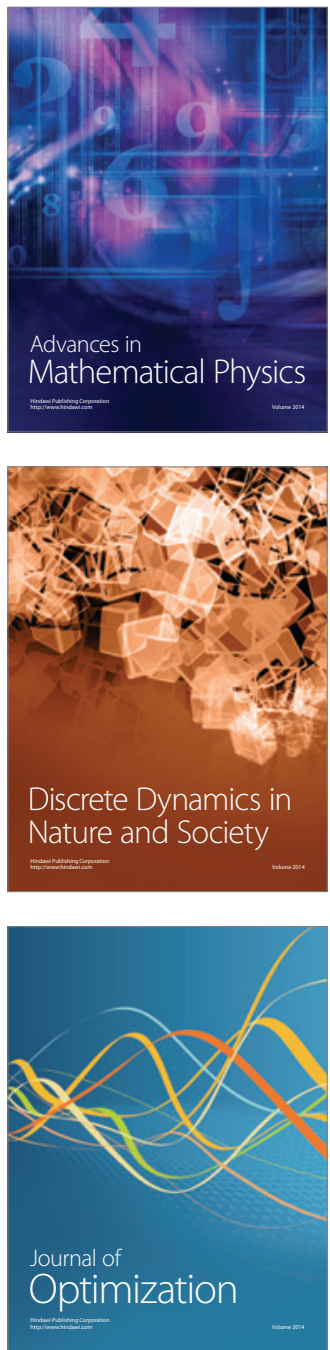